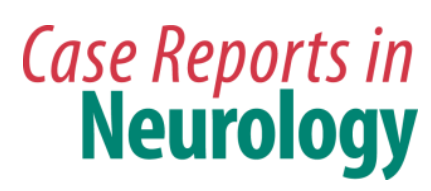

Case Rep Neurol 2014;6:1-6

\title{
Essential Tremor in a Charcot-Marie-Tooth Type 2C Kindred Does Not Segregate with the TRPV4 R269H Mutation
}

\author{
Elan D. Louis ${ }^{a-c, e} \quad$ Nora Hernandez ${ }^{a}$ Ruth Ottman ${ }^{a, c, e, h}$ \\ Iuliana Ionita-Laza ${ }^{f}$ Lorraine N. Clark ${ }^{\mathrm{b}, \mathrm{d}}$ \\ ${ }^{a}$ G.H. Sergievsky Center, ${ }^{b}$ Taub Institute for Research on Alzheimer's Disease and the \\ Aging Brain, and Departments of ${ }^{c}$ Neurology and ${ }^{d}$ Pathology and Cell Biology, College of \\ Physicians and Surgeons, Columbia University, Departments of ${ }^{\mathrm{e}}$ Epidemiology and \\ fBiostatistics, Mailman School of Public Health, Columbia University, ${ }^{9}$ Department of \\ Pathology and Cell Biology, Columbia University Medical Center, and hivision of \\ Epidemiology, New York State Psychiatric Institute, New York, N.Y., USA
}

\section{Key Words}

Essential tremor · Charcot-Marie-Tooth · Neuropathy · Genetics

\begin{abstract}
Background: We investigated 4 members of a family with type 2C Charcot-Marie-Tooth (CMT) and self-reported essential tremor (ET). A heterozygous missense mutation, $\mathrm{R} 269 \mathrm{H}$, in the TRPV4 gene was previously reported in this family. Our genotypic data provided a rare opportunity to determine the etiology of the tremor. Methods: Family study; the 4 tremor cases underwent a detailed neurological assessment. Results: The clinical diagnosis of ET was confirmed in all 4 tremor cases based on stringent published research criteria. Two of these also had CMT. We genotyped all 4 family members for the TRPV4 R269H mutation. We confirmed the presence of the TRPV4 R269H mutation in the 2 family members with ET and CMT; however, the TRPV4 R269H mutation did not segregate with ET in the same family. Conclusions: In this particular CMT family, the tremor was clinically attributed to ET. Furthermore, genotype data indicated that the tremor was unlikely to be caused by incomplete penetrance or variable expressivity of the TRPV4 R269H mutation. Hence, the tremor likely represents ET. This establishes that in some CMT families the tremor diathesis likely represents a second disorder, namely ET.


Louis et al.: Essential Tremor in a Charcot-Marie-Tooth Type 2C Kindred Does Not Segregate with the TRPV4 R269H Mutation

\section{Introduction}

Several neuropathies are accompanied by action tremors [1-4]. Charcot-Marie-Tooth (CMT) disease (hereditary sensory and motor neuropathy) is a heterogeneous group of familial neuropathies in which tremor is reported to be common, but the tremor is not well defined $[5,6]$. It remains a matter of debate as to whether this tremor is a phenotypic expression of CMT or whether it represents a comorbidity [i.e. essential tremor (ET)] [5, 6]. Indeed, the diagnostic term 'essential tremor' has been applied repeatedly to many of these cases [6].

We investigated a family with type 2C CMT (associated especially with lower limb and voice abnormalities) and self-reported ET; the family was enrolled in the Family Study of Essential Tremor (FASET), Columbia University Medical Center (CUMC) [7]. The genotypic data provided a rare opportunity to attempt to disentangle the etiology of the tremor.

Several members of the kindred have been reported by other investigators who focused on neuropathy rather than tremor [8,9]; a heterozygous missense mutation, $\mathrm{R} 269 \mathrm{H}$, in the TRPV4 gene was identified [9]. We genotyped 4 family members with mixed phenotypes (2 family members had been diagnosed with ET, and 2 others had been diagnosed with both ET and CMT) for the TRPV4 R269H mutation, and present our results.

\section{Methods}

\section{Subjects/Evaluation}

The family was enrolled in a genetic study of ET, as described [7, 10]. The study was approved by the CUMC Institutional Review Board; participants gave written informed consent. Seven individuals in the family were enrolled.

An in-person evaluation was conducted (questionnaires, videotaped neurological examination [7], and phlebotomy). The videotaped neurological examination included detailed assessments of postural, kinetic, intention and rest tremors, dystonia, and other movement disorders [11]. E.D.L. reviewed these examinations and rated the severity of postural and kinetic arm tremors (ratings of $0-3$ in 12 items), resulting in a total tremor score [range $=0$ 36 (maximum)] [11]. All ET diagnoses were reconfirmed based on a review of questionnaires and videotaped neurological examinations [7]. Diagnoses were assigned based on stringent published diagnostic criteria (moderate or greater amplitude kinetic tremor during $\geq 3$ activities or a head tremor in the absence of Parkinson's disease or another known cause) [12]. The presence of dystonic postures did not preclude the diagnosis, particularly if mild and different in distribution from the tremor (e.g. blepharospasm).

\section{Genotyping of TRPV4 Mutations in the CMT Type 2C Family by Sanger Sequencing}

Genomic DNA was isolated from peripheral blood using the DNeasy blood kit (Qiagen, Inc.) or from saliva using the Oragene DNA kit (DNA Genotek Inc.). TRPV4 mutations were sequenced by means of polymerase chain reaction and using sequencing primers described previously [9]. Cycle sequencing in forward and reverse directions was performed on purified polymerase chain reaction products and run on a genetic analyzer (ABI 3700; Applied Biosystems, Foster City, Calif., USA). Sequence chromatograms were viewed and genotypes determined by means of Sequencher (Gene Codes Corp, Ann Arbor, Mich., USA). 
Louis et al.: Essential Tremor in a Charcot-Marie-Tooth Type 2C Kindred Does Not Segregate with the TRPV4 R269H Mutation

\section{Results}

\section{Phenotypes}

The proband, subject III.6, was a 61-year-old woman whose tremor began at age 30 (table 1). On examination, there was a mild bilateral postural tremor and moderate bilateral kinetic tremor during multiple activities (total tremor score $=20$ ). There was no intention or rest tremor. There was mild side-to-side neck tremor, and no limb or cranial dystonia or parkinsonism (fig. 1; table 1). The subject had been diagnosed with CMT at age 37 but had been symptomatic for 1-2 years with leg weakness and changes in the quality of her voice, as is typical of CMT type 2C. An electromyogram/nerve conduction study at age 36 was consistent with a predominantly axonal peripheral neuropathy involving both upper and lower extremities.

Subject III.2, her brother, was a 72-year-old man. On examination, there was a mild bilateral postural tremor and moderate bilateral kinetic tremor during multiple activities (total tremor score $=20$, online suppl. fig. 1 ; for all online suppl. material, see www.karger.com/doi/10.1159/000357665). There was no intention or rest tremor. There was subtle flexed posturing of the left wrist during arm extension. There was no head tremor and no cranial dystonia or parkinsonism (table 1). The patient was unable to recall the age of onset of tremor or date of CMT diagnosis. There was bilateral arm and leg weakness with wasting of intrinsic hand muscles; the patient ambulated with a walker.

Subject III.4, their sister, was 81 years old. On examination, there was a mild left arm postural tremor, mild right arm kinetic tremor, moderate left arm kinetic tremor (total tremor score $=16$, table 1 ), jaw tremor and subtle blepharospasm. She did not have CMT.

Subject II.5, their maternal uncle, was an 87-year-old man with tremor onset at age 23 (online suppl. fig. 2; table 1). He had moderate to severe postural and kinetic tremor (total tremor score $=31$ ), with an intentional component bilaterally. Aside from a mild bilateral rest tremor, there were no features of parkinsonism. He did not have CMT.

\section{Identification of TRPV4 Mutations}

To check cosegregation of the TRPV4 mutations in family members with CMT type 2C and ET, we performed Sanger sequencing in the 4 family members (II.5, III.2, III.4, and III.6) and 3 other family members (III.8, III.10, and III.11) with available DNA. We confirmed the presence of the TRPV4 R269H mutation in family members III.2 and III.6 (fig. 1; online suppl. fig. 3). Neither family members II.5 and III.4 (both with a diagnosis of ET) nor III.8 and III.11 (normal) carried the TRPV4 R269H mutation. Subject III.10 with CMT type 2C and ET carried the TRPV4 R269H mutation (fig. 1).

\section{Discussion}

The tremor in CMT patients remains a source of diagnostic confusion for clinicians and a potential source of error in genetic studies of ET. It is more parsimonious to attribute the tremor to one disorder, CMT, than to invoke comorbidity with ET. Yet our family provides firm support that the tremor, at least in some families, is not merely the result of CMT. It is not known whether CMT and ET, as 2 disease entities, are associated with one another at the population level. Ours was a kindred study; epidemiological studies have not been conducted to address this specific question.

Two of our cases (III.2 and III.4) had very subtle and focal dystonia on examination; in both cases, the dystonia was isolated to 1 limb or 1 cranial structure, was asymptomatic, and 
not diagnosed by their physician. The tremor was the predominant clinical feature and was present in numerous body regions that dystonia was not. Dystonia, of varying severity, may be a feature in some ET families [7]. Furthermore, the presence of mild dystonia, particularly in the presence of long-standing or severe tremor, does not preclude the diagnosis of ET (Consensus Meeting of the Tremor Task Force of the Movement Disorders Society, Lisbon, May 3-5, 2013, pers. commun.).

In summary, we confirmed the presence of the TRPV4 R269H mutation in the 2 family members with ET and CMT; however, 2 family members with ET in the absence of CMT did not have the TRPV4 R269H mutation. This suggests that tremor in this family is unlikely to be an expression of CMT and unlikely to represent incomplete penetrance or variable expressivity of the TRPV4 R269H mutation. The tremor likely represents ET, as diagnosed clinically. Follow-up studies are underway to identify the causal mutation for ET in this family.

\section{Disclosure Statement}

The authors declare that there are no conflicts of interest and no competing financial interests. This research was supported by National Institutes of Health grant R01 NS073872.

\section{References}

1 Budak F, Alemdar M, Kamaci S, Selekler M: Tremor in idiopathic distal acquired demyelinating symmetric neuropathy. Mov Disord 2005;20:1529-1530.

-2 Pedersen SF, Pullman SL, Latov N, Brannagan TH 3rd: Physiological tremor analysis of patients with antimyelin-associated glycoprotein associated neuropathy and tremor. Muscle Nerve 1997;20:38-44.

-3 Kamei H, Nishimaru K, Abe H, Irie M, Ohnishi A: A case of hereditary motor and sensory neuropathy (HMSN type 2) with bilateral recurrent nerve palsy (in Japanese). Rinsho Shinkeigaku 1993;33:957-960.

$\checkmark 4$ Saverino A, Solaro C, Capello E, Trompetto C, Abbruzzese G, Schenone A: Tremor associated with benign IgM paraproteinaemic neuropathy successfully treated with gabapentin. Mov Disord 2001;16:967-968.

-5 Cardoso FE, Jankovic J: Hereditary motor-sensory neuropathy and movement disorders. Muscle Nerve 1993;16:904-910.

-6 Salisachs P: Charcot-Marie-Tooth disease associated with 'essential tremor': report of 7 cases and a review of the literature. J Neurol Sci 1976;28:17-40.

7 Louis ED, Hernandez N, Alcalay RN, Tirri DJ, Ottman R, Clark LN: Prevalence and features of unreported dystonia in a family study of 'pure' essential tremor. Parkinsonism Relat Disord 2013;19:359-362.

-8 Dyck PJ, Litchy WJ, Minnerath S, Bird TD, Chance PF, Schaid DJ, Aronson AE: Hereditary motor and sensory neuropathy with diaphragm and vocal cord paresis. Ann Neurol 1994;35:608-615.

-9 Landoure G, Zdebik AA, Martinez TL, Burnett BG, Stanescu HC, Inada H, Shi Y, Taye AA, Kong L, Munns CH, Choo SS, Phelps CB, Paudel R, Houlden H, Ludlow CL, Caterina MJ, Gaudet R, Kleta R, Fischbeck KH, Sumner CJ: Mutations in TRPV4 cause Charcot-Marie-Tooth disease type 2C. Nat Genet 2010;42:170-174.

$\rightarrow 10$ Louis ED, Hernandez N, Ionita-Laza I, Ottman R, Clark LN: Does rate of progression run in essential tremor families? Slower vs. Faster progressors. Parkinsonism Relat Disord 2013;40:269-273.

11 Louis ED, Jiang W, Pellegrino KM, Rios E, Factor-Litvak P, Henchcliffe C, Zheng W: Elevated blood harmane (1-methyl-9h-pyrido[3,4-b]indole) concentrations in essential tremor. Neurotoxicology 2008;29:294-300.

12 Louis ED, Ford B, Bismuth B: Reliability between two observers using a protocol for diagnosing essential tremor. Mov Disord 1998;13:287-293. 


\begin{tabular}{|c|c|}
\hline \multicolumn{2}{|l|}{ Case Rep Neurol 2014;6:1-6 } \\
\hline DOI: 10.1159/000357665 & $\begin{array}{l}\text { (C) } 2014 \text { S. Karger AG, Basel } \\
\text { www.karger.com/crn }\end{array}$ \\
\hline
\end{tabular}

Louis et al.: Essential Tremor in a Charcot-Marie-Tooth Type 2C Kindred Does Not Segregate with the TRPV4 R269H Mutation

Table 1. Demographic and clinical features of 4 family members

\begin{tabular}{|c|c|c|c|c|}
\hline & III.6 & III. 2 & III.4 & II.5 \\
\hline Current age, years & 61 & 72 & 81 & 87 \\
\hline Right- or left-handed & Right & Right & Right & Right \\
\hline Gender & Female & Male & Female & Male \\
\hline Age at tremor onset, years & 30 & Could not recall & 65 & 23 \\
\hline Previously diagnosed with ET by a physician & Yes & No & Yes & Yes \\
\hline Prescribed ET medication & Yes & No & No & Yes \\
\hline Symptomatic improvement with ET medication & Yes & No & No & Yes \\
\hline Previously diagnosed with dystonia by a physician & No & No & No & No \\
\hline Previously diagnosed with CMT & Yes & Yes & No & No \\
\hline Diagnosed with ET based on current assessment & Yes & Yes & Yes & Yes \\
\hline \multicolumn{5}{|l|}{ Examination } \\
\hline Extension (R) ${ }^{1}$ & 1 & 1 & 0 & 2 \\
\hline Extension $(\mathrm{L})^{1}$ & 1 & 1 & 1 & 3 \\
\hline Pouring (R) ${ }^{1}$ & 2 & 1 & 1 & 2 \\
\hline Pouring (L) ${ }^{1}$ & 2 & 2 & 2 & 2 \\
\hline Using spoon $(R)^{1}$ & 2 & 2 & 1 & 3 \\
\hline Using spoon (L) ${ }^{1}$ & 3 & 2 & 2 & 3 \\
\hline Drinking $(\mathrm{R})^{11}$ & 2 & 1 & 1 & 2 \\
\hline Drinking (L) ${ }^{1}$ & 2 & 2 & 2 & 3 \\
\hline Finger-nose-finger $(\mathrm{R})^{1}$ & 1 & 2 & 1 & 2 \\
\hline Finger-nose-finger $(\mathrm{L})^{1}$ & 2 & 2 & 2 & 3 \\
\hline Archimedes spiral (R) ${ }^{1}$ & 1 & 2 & 1 & 3 \\
\hline Archimedes spiral (L) ${ }^{1}$ & 1 & 2 & 2 & 3 \\
\hline Total tremor score & 20 & 20 & 16 & 31 \\
\hline Neck tremor & Yes & No & No & No \\
\hline Jaw tremor & No & No & Yes & Yes \\
\hline Voice tremor & No & No & No & No \\
\hline Dystonia & No & Yes (left arm) & Yes (blepharospasm) & No \\
\hline Parkinsonism & No & No & No & No \\
\hline Limb weakness & Yes (arms, legs) & Yes (arm, legs) & No & No \\
\hline Areflexia & Yes & Yes & No & No \\
\hline
\end{tabular}

R = Right; L = left. ${ }^{1}$ Washington Heights Inwood Genetic Study of Essential Tremor ratings (range $=0-3$ ). 
:

II

Louis et al.: Essential Tremor in a Charcot-Marie-Tooth Type 2C Kindred Does Not



Fig. 1. Pedigree of family and electropherograms showing clinical diagnosis of CMT or ET and family members who carry the TRPV4 mutation. The proband is denoted by an arrow. Squares and circles denote males and females, respectively, and diagonal lines represent deceased individuals. Black squares inside of grey shading represent family members diagnosed with CMT and ET, black squares without gray shading represent family members diagnosed with ET only, and gray shading without black squares represents family members diagnosed with CMT only, and open symbols indicate unaffected individuals. M = TRPV4 c.806G>A (p.R269H); W = wild-type allele. 